\section{Embedded power plane with ultra-wide stop-band for simultaneously switching noise on high-speed circuits}

\author{
T.L. Wu and T.K. Wang
}

An embedded band selective (EBS) power plane is proposed using the hybrid-cell periodic structure. Because the periodic connections of the unit-hybrid-cell select different frequency rejection bands, the proposed EBS power plane performs ultra-wideband suppression of the simultaneously switching noise (about $9 \mathrm{GHz}$ ) with on average, over $60 \mathrm{~dB}$ noise elimination. This excellent behaviour is both numerically and experimentally validated.

Introduction: Transient current surges resulting from the simultaneous switching of output buffers in high-speed integrated circuits can create significant ground bounce noise (GBN) or simultaneously switching noise (SSN) on the chip, package, and printed circuit board (PCB) [1-3]. The SSN causes signal integrity (SI) problems, such as glitches or timing push-out of signal traces, and electromagnetic interference (EMI) issues in high-speed circuits [3]. With the trend to increase clock speed and the drop of the power supply voltage for high-speed circuits, elimination of the SSN is becoming important.

Adding decoupling capacitors between the power and ground planes is a typical way to suppress the SSN. However, in general, these capacitors are not effective at frequencies higher than $600 \mathrm{MHz}$ owing to finite lead inductance. Recently, a new idea for eliminating the SSN is proposed by designing an electromagnetic bandgap (EBG) structure on the ground or power plane [4-6]. Because the SSN is distributed over a wide frequency range from $\mathrm{MHz}$ to several $\mathrm{GHz}$ [4], several new EBG power/ground plane designs have been proposed to broaden the stop-band bandwidth for suppressing the SSN. The concept of cascading EBG structures with different stop-bands employing the inductance-enhanced high impedance surface (HIS) were presented to achieve wider bandgap bandwidth $[4,5]$, but there are some drawbacks. One is the substantial increase in fabrication cost because three or four layers of metal are needed for implementing the HIS and much more power/ground plane area is occupied to cascade different stop-band EBGs. The other is that performance is degraded at the transition frequency range between the two stop-bands for the cascading design.

This Letter proposes a novel embedded band selective (EBS) power plane with over $9 \mathrm{GHz}$ stop-band using a hybrid-cell periodic structure (HCPS). The key feature of the proposed power/ground plane is keeping the ground plane continuous and designing the HCPS on the power plane. The advantages of the EBS power/ground plane are ultra-wide stop-band for the GBN, low cost because only two metal layers are enough to achieve the SSN suppression, easy fabrication owing to compatible to the conventional package or $\mathrm{PCB}$ manufacturing process.
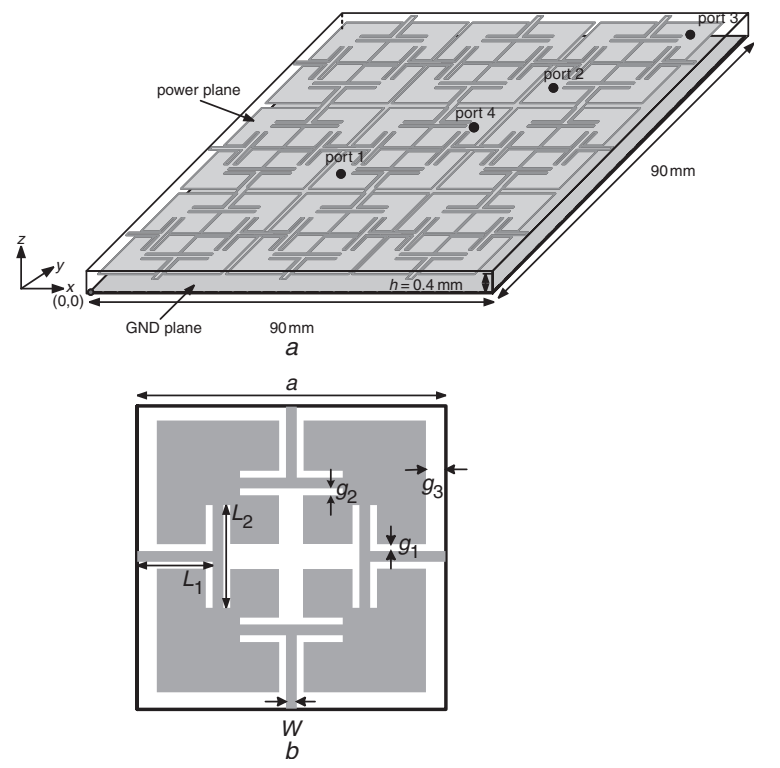

Fig. 1 Proposed design and unit-hybrid-cell

$a$ Proposed EBS power/ground plane design

$b$ Unit-hybrid-cell and its corresponding parameter notations
Design concept: Fig. 1a shows the proposed EBS power/ground plane design. The ground plane is kept continuous and the HCPS is designed on the power plane with nine hybrid cells. The unit-hybridcell and its corresponding parameter notations are shown in Fig. $1 b$. The square unit-hybrid-cell consists of four sub-cells. Each sub-cell has equal area and electrically connects to neighbouring sub-cells with a short bridge. The power plane is constructed by repeating nine $(3 \times 3)$ unit hybrid cells, and the adjacent hybrid cells are also electrically connected with a bridge. The band stop characteristic on the power and ground planes can be achieved by the distributed LC networks realised by the etched metal pads on the power plane and the narrow lines connecting between the pads. There are two different distributed LC networks; one consists of the pads of the unit-hybridcells and the bridge between them and the other consists of the pads of the sub-cells and the shorter bridge between sub-cells. These two networks select different frequency ranges for band rejection and, therefore, contribute to the ultra-wide suppression of the GBN. The design parameters, denoted in Fig. $1 b$, are $w=0.6 \mathrm{~mm}$, $L_{1}=7.125 \mathrm{~mm}, L_{2}=10.8 \mathrm{~mm}, g_{1}=g_{2}=0.6 \mathrm{~mm}, g_{3}=0.75 \mathrm{~mm}$ on the PCB substrate $0.4 \mathrm{~mm}$ thick. The dimensions of the PCB are $9 \times 9 \mathrm{~cm}$.

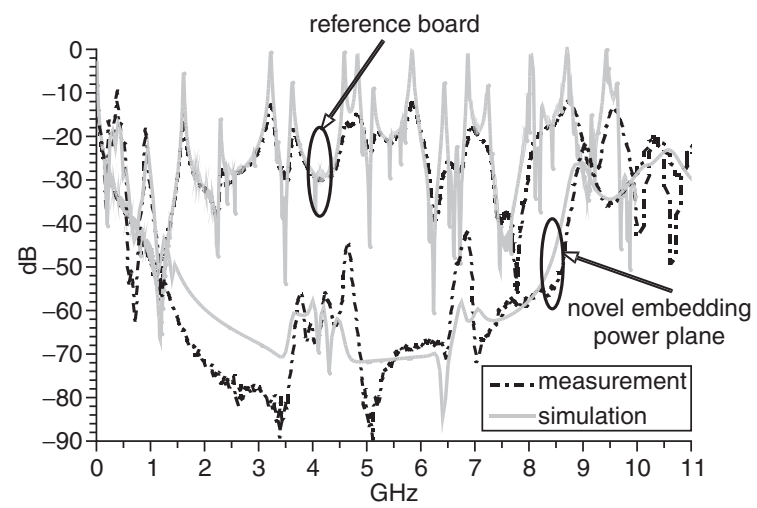

Fig. 2 Measured and simulated $\left|S_{21}\right|$ for designed EBS power/ground plane

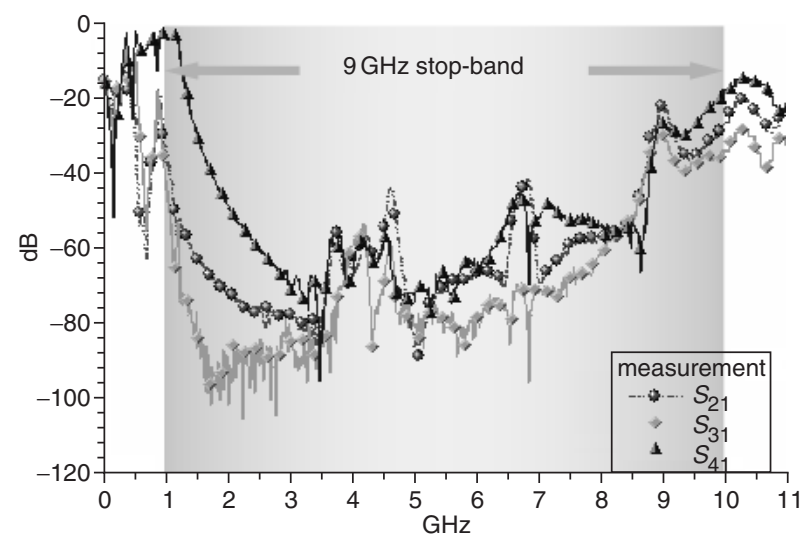

Fig. 3 Measured SSN suppression behaviour for noise excitation located at different locations; port 2 , port 3 , and port 4 , respectively

Results: A prototype of the designed EBS power/ground plane using the previous parameters has been fabricated and measured. Fig. 2 shows the measured and simulated $\left|S_{21}\right|$ for the designed EBS power/ground plane. The insertion loss of the reference board with both power and ground planes being solid is also presented in this Figure for comparison. The EM tool HFSS is used to simulate the SSN behaviour of the structure. Excellent agreement between measurement and simulation from DC to $11 \mathrm{GHz}$ is seen. Because the dispersion property of the FR4 substrate is not considered in the modelling, slight difference between them is seen at higher frequencies above $8 \mathrm{GHz}$. Compared with the reference board, it is clearly seen that the proposed EBS power plane gives highly efficient SSN elimination with, on average, $60 \mathrm{~dB}$ suppression in a wide-band range from about 1 to $10 \mathrm{GHz}$ ( $9 \mathrm{GHz}$ bandwidth). The bandwidth is defined by the insertion loss lower than $-20 \mathrm{~dB}$. 
Fig. 3 shows the measured SSN suppression behaviour for the noise excitation located at different locations; port 2, port 3, and port 4, respectively. The receiving port is all at port1. It is seen that the broadband SSN suppression behaviour is almost the same for different excitation locations of excitation. It implies that the novel EBS power plane can omnidirectionally eliminate the SSN on the power/ground plane.

Conclusion: A novel embedded band selective (EBS) power/ground plane is proposed using the hybrid-cell periodic structure. Because the periodic connections of the hybrid-cell select different frequency rejection bands, the proposed EBS power plane performs ultra-wideband suppression of the GBN (about $9 \mathrm{GHz}$ ) with, on average, over $60 \mathrm{~dB}$ noise elimination. This excellent behaviour is validated both by simulation and measurement.

Acknowledgments: This work is supported by the National Science Council (NSC) under grant number NSC93-2213-E-110-010.

\section{(C) IEE 2006}

3 October 2005

Electronics Letters online no: 20063498

doi: 10.1049/el:20063498

T.L. Wu and T.K. Wang (Department of Electrical Engineering and Graduate Institute of Communication Engineering, National Taiwan University, Taipei, Taiwan)

E-mail: wtl@cc.ee.ntu.edu.tw

\section{References}

1 Van den Berghe, S., Olyslager, F., De Zutter, D., De Moerloose, J., and Temmerman, W.: 'Study of the ground bounce caused by power plane resonances', IEEE Trans. Electromagn. Compat., 1998, 40, (2), pp. 111-119

2 Lei, G., Techentin, R.W., and Bilbert, B.K.: 'High-frequency characterization of power/ground-plane structures', IEEE Trans. Microw. Theory Tech., 1999, 47, pp. 562-569

3 Wu, T.L., Chen, S.T., Huang, J.N., and Lin, Y.H.: 'Numerical and experimental investigation of radiation caused by the switching noise on the partitioned DC reference planes of high speed digital PCB', IEEE Trans. Electromagn. Compat., 2004, 46, pp. 33-45

4 Abhari, R., and Eleftheriades, G.V.: 'Metallo-dielectric electromagnetic bandgap structures for suppression and isolation of the parallel-plate noise in high-speed circuits', IEEE Trans. Microw. Theory Tech., 2003, 51, (6), pp. 1629-1639

5 Kamgaing, T., and Ramahi, O.M.: 'A novel power plane with integrated simultaneous switching noise mitigation capability using high impedance surface', IEEE Microw. Wirel. Compon. Lett., 2003, 13, (1), pp. 21-23

6 Wu, T.L., Lin, Y.H., and Chen, S.T.: 'A novel power plane with low radiation and broadband suppression of ground bounce noise using photonic bandgap structures', IEEE Microw. Wirel. Compon. Lett., 2004, 14, (7), pp. 337-339 